\title{
Dynamics of endoscopic snares: a new approach towards more practical and objective performance evaluation $\square$
}

\section{다 (1) $\odot$}

\author{
Authors \\ Masaomi Agatsuma ${ }^{5}$, Hiroyuki Okada', Kiyokazu Nakajima3,4 \\ Institutions \\ 1 Department of Gastroenterology, Okayama University \\ Hospital, Okayama, Japan \\ 2 Department of Gastrointestinal Oncology, Osaka \\ International Cancer Institute, Osaka, Japan \\ 3 Department of Next Generation Endoscopic \\ Intervention (Project ENGINE), Osaka University \\ Graduate School of Medicine, Osaka, Japan \\ 4 Department of Gastroenterological Surgery, Osaka \\ University Graduate School of Medicine, Osaka, Japan \\ 5 Hakko Co., Ltd., Nagano, Japan
}

Yasushi Yamasaki ${ }^{1,2}$, Yoji Takeuchi ${ }^{2}$, Yuki Ushimaru ${ }^{3,4}$, Noriko Matsuura², Tsuyoshi Yamaguchi ${ }^{5}$, Shun Nitta $^{5}$,

submitted 25.11.2019

accepted after revision 2.3.2020
Bibliography

DOI https://doi.org/10.1055/a-1145-3308 |

Endoscopy International Open 2020; 08: E792-E795

(c) Georg Thieme Verlag KG Stuttgart · New York elSSN 2196-9736

Corresponding author

Kiyokazu Nakajima, Department of Next Generation Endoscopic Intervention (Project ENGINE), Osaka University Graduate School of Medicine, Suite 0912, 2-2, Yamadaoka, Suita, Osaka, 565-0871, Japan

Fax: +81-6-6210-8420

knakajima@gesurg.med.osaka-u.ac.jp
Endoscopic resection using polypectomy snares is a standard method for treating gastrointestinal polyps. However, because few systems can objectively evaluate the capabilities of these snares, endoscopists tend to use them according to their personal preference. We developed a novel objective experimental system to evaluate the dynamic performance of these snares and evaluated their feasibility and reproducibility.

Using our system, we measured the sequential changes in hold-down and tightening force, which are essential capacities for all resection techniques using snares. To evaluate the system's feasibility, the dynamic performances of three representative snares (product $A$ : an oval-shaped standard snare, product B: a hexagonal-shaped standard snare, and product C: a rounded hard snare) were measured repeatedly.

Forces of each snare were measured to objectively show the dynamic changes in hold-down force during snaring. Results for each time point showed good reproducibility. The forces of product $C$ were larger than those of products $A$ and $B$, reflecting the subjective estimation on the market of the commercially available snares.

We developed a novel system for objectively measuring the dynamic performance of polypectomy snares. This system warrants further experiments.

\section{Introduction}

Endoscopic resection using polypectomy snares is a common, standard technique for treating gastrointestinal polyps [1-3]. Several safe and effective resection methods are available, including endoscopic mucosal resection, cold snare polypectomy, hot snare polypectomy, and underwater endoscopic mucosal resection [4-6]. Accordingly, various commercially available polypectomy snares have been developed.

Theoretically, the "right tool" for each lesion can be selected, according to the procedure and lesion characteristics (e.g., size, shape and location). For example, sustainable hold-down force is desirable if a polyp is located at the colonic flexure, and sharp cutting force is desirable for cold snare polypectomy [7]. In the real world, however, endoscopists must choose each snare according to personal preference, primarily because few methods exist to objectively compare each device's performance [8]. The previously reported methodology only evaluated the "static" performance in a purely experimental setting [8], although fold-down and tightening forces can change dynamically during snaring. A more practical method for better understanding the "dynamic" performance of polypectomy snares may facilitate their logical choice and right use.

Thus, we developed a new, more practical, dynamic method to evaluate the performance of polypectomy snares. This study was conducted to show the feasibility and reproducibility of our new objective evaluation method. 


\section{Methods}

We designed a new experimental system to evaluate the capabilities and measure the dynamic parameters of polypectomy snares. Our experimental system objectively showed sequential change in hold-down force (i.e., a change in the horizontal-facing position weight, shown as follows) and tightening force. Details and representative data for the snares obtained via the new system are described herein.

\section{Novel system to measure dynamic parameters}

$>$ Fig. 1a, > Fig. 1b and $>$ Video 1 show the system for measuring sequential changes in hold-down force. Before the experiment, a snare was introduced into the working channel of an endoscope. The snare handle was then set on a horizontal compatible-type electric stand (Model-2257, Aikoh Engineering Co., Ltd.), and the finger rings were fixed. The tip of the snare was placed on the saucer of the digital push-pull gauge (RX-10, Aikoh Engineering Co., Ltd.), with the incidence angle toward the push-pull gauge maintained at 20 degrees ( Fig.1a). At this stage, the snare sheath was fixed $10 \mathrm{~mm}$ from the tip of the endoscope. The scope was then moved downward, and the fully opened snare was placed on the flat saucer of the gauge with the whole loop facing horizontally. Next, the snare was closed at a constant speed $(100 \mathrm{~mm} / \mathrm{min}$;

- Fig.1b), and the sequential change in horizontal position weight, which was defined as the dynamic change in holddown force, was recorded.

\section{Data calculation}

Sequential changes in horizontal position weight (dynamic change in hold-down force) are shown in a line graph. Gradients of the graph [= $\Delta$ horizontal position weight (gf) $/ \Delta$ moving distance $(\mathrm{mm})]$ at the snare starting and closing were defined as the tightening force (red arrow in $>$ Fig. 2 a, > Fig. $\mathbf{2 b}$ and - Fig. 2c). Using the aforementioned methodology, dynamic data were measured seven times for the three snare types in the same setting (see results section). One polypectomy snare was repeatedly used in each experiment. Each time is shown as a different color in the line graph, and each snare gradient is shown as the mean value from seven recordings. Product A (a 15-mm Snare master, SD-210U-15, Olympus, Tokyo, Japan) was an oval-shaped standard capability snare, which was subjectively recognized to have standard fold-down and tightening force on the market. Product B (a 15-mm Snare master plus, SD-400U-15, Olympus, Tokyo, Japan) was a modified hexagonal-shaped snare made of thin wire for easy snaring. Product $C$ (a 15-mm Captivator II, M00561230, Boston Scientific, Marlborough, Massachusetts, United States) was a hard, rounded snare, which was subjectively recognized to have strong folddown and tightening force on the market.

\section{Results}

$>$ Fig. 2a, > Fig. 2b and > Fig. 2c show each snare's recorded dynamic parameters. Horizontal position weight (hold-down force) of product $A$ increased gradually and shows as low gradi-

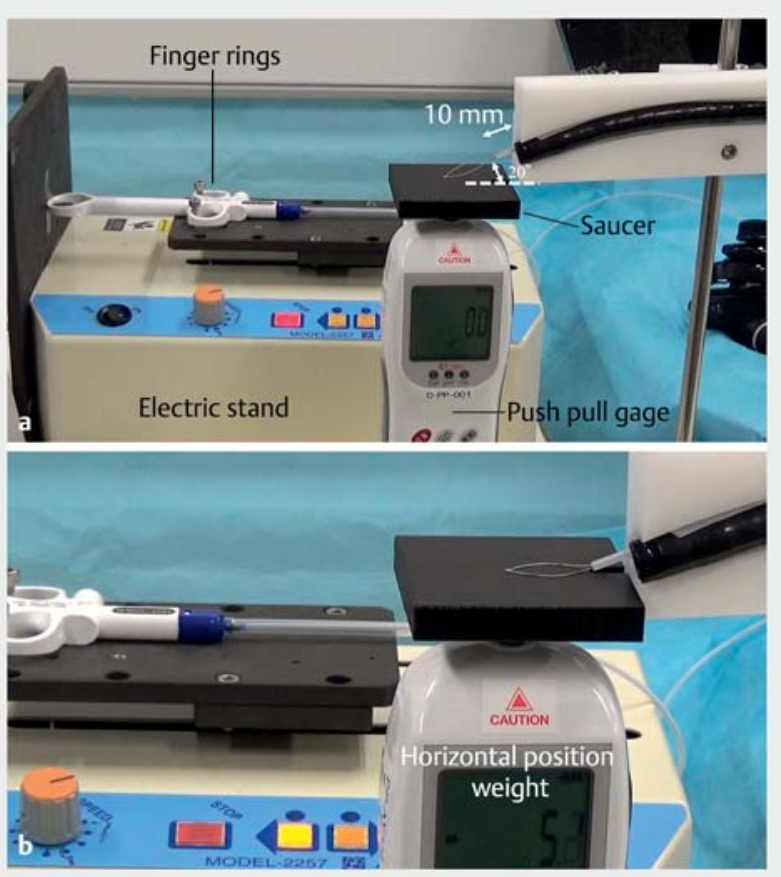

- Fig. 1 Dynamic system for measuring sequential change in hold-down force. a Snare setting before measurement. b Change in hold-down force was measured chronologically.

ents (mean value \pm standard deviation, $0.27 \pm 0.008 \mathrm{gf} / \mathrm{mm}$, defined as the tightening force) as the snare closed. Maximum horizontal position weight is shown at the end ( $\mathbf{F i g . 2 a )}$. Conversely, horizontal position weight of product $B$ increased rapidly and shows as high gradients (mean value \pm standard deviation, $1.13 \pm 0.07 \mathrm{gf} / \mathrm{mm}$ ), with the moderate horizontal position weight being maintained. Horizontal position weight of product $C$ also increased rapidly from the start of the snare

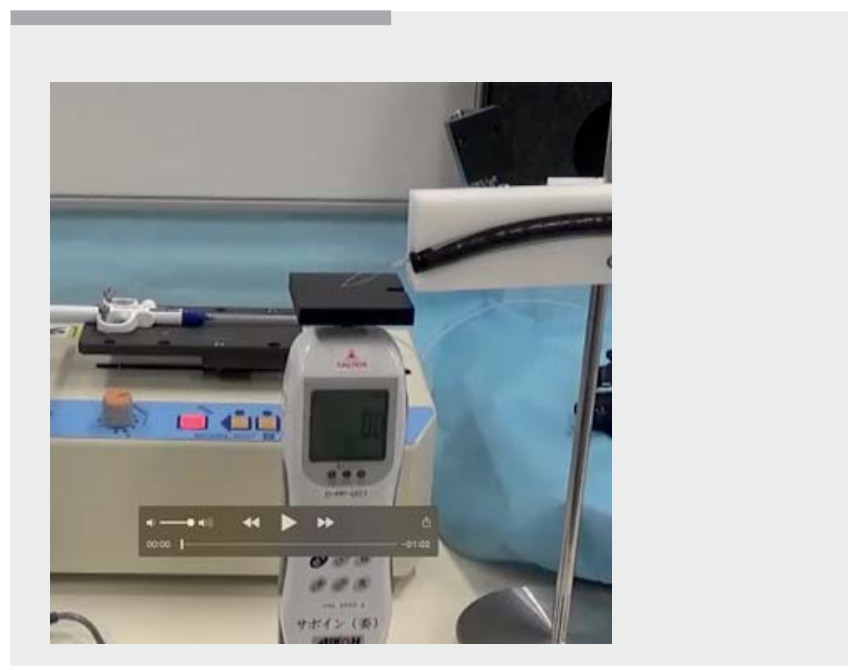

Video 1 Novel system to measure the dynamics of polypectomy snares. The video shows the method of measuring the dynamic parameters. 

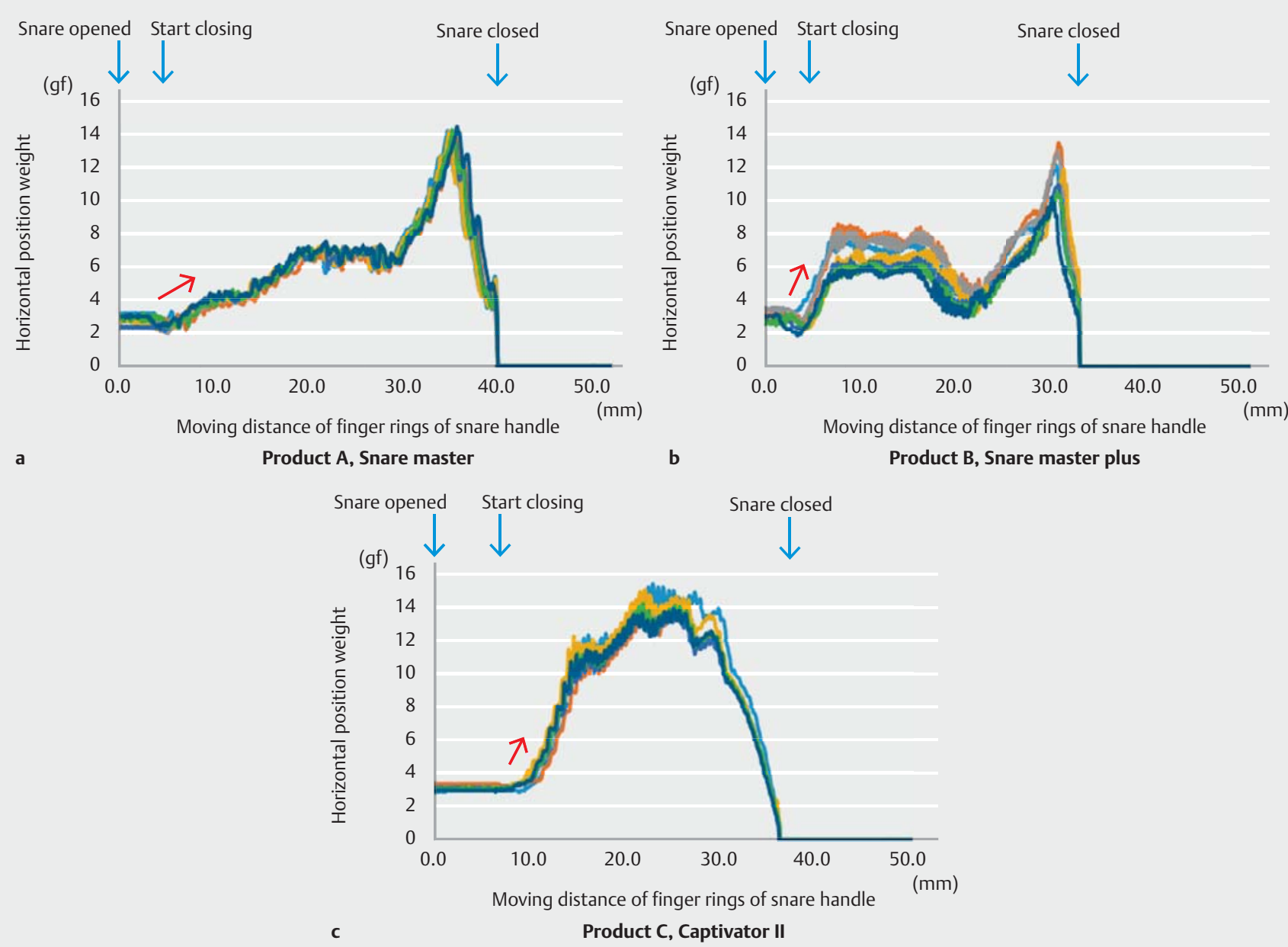

- Fig. 2 Representative data measured by our system. Dynamic performance of each snare was measured seven times and is shown as a line graph. Red arrows are gradients. a Line graph of product A (oval standard snare). b Line graph of product B (hexagonal standard snare). c Line graph of product C (round hard snare). Notes: "Snare opened" was defined as the snare being fully opened, and the opening movement of the handle was complete. "Start closing" was defined as the point when the fully opened snare started closing. "Snare closed" was defined as the snare being fully closed, and the closing movement of the handle was complete.

closing and shows as high gradients (mean value \pm standard deviation, $1.38 \pm 0.10 \mathrm{gf} / \mathrm{mm}$ ), with the large horizontal position weight being maintained ( $\boldsymbol{F}$ Fig. $2 \mathbf{c}$ ). All snare parameters were similar in each measurement; thus, the fold-down and tightening force are reproducible.

\section{Discussion}

We developed a novel experimental system to evaluate dynamics of three polypectomy snares and described usage and data obtained from the system. Here, we objectively showed for the first time the system's snare dynamics, and this Innovation Forum article shows our system's reproducibility.

Each snare should be evaluated objectively before applying it in vivo. "Static" evaluation via bench testing and usability in animal models have been assessed previously [8]; however, these tests were insufficient to fully evaluate the snares. Each snare's performance would change during snaring; thus, "static" data may change according to the measured points, even if each snare is evaluated similarly. Animal models provide useful information for endoscopists; however, usability in animal models is subjective rather than objective, and the snare's performance in animal models is not always transferrable to humans. Furthermore, excessive animal experimentation violates the terms of the Declaration of Helsinki $[9,10]$. Therefore, objective and dynamic bench-test evaluations, which can reflect snare performance in vivo, are essential. Thus, we developed an objective and "dynamic" experimental system and assessed its feasibility.

In our system, sequential changes in hold-down and tightening force were measured because they are essential capacities for all resection techniques that use snares. Sufficient holddown force is required to fix the opened snare at the normal mucosa around the polyp [8], and sustained hold-down force is required to continuously capture the polyp during the snaring procedure (from capture until the polyp is resected). Further, adequate tightening force is required to prevent the snare from slipping out of the captured mucosa during the proce- 
dure. We measured these objective parameters for three commercially available snares to enable comparing the snares considering subjective information on the market. The hard snare (product C), which was recognized to have the greatest subjective hold-down and tightening force on the market, showed greater objective hold-down and tightening force than the standard snares (products $A$ and $B$ ). Products $A$ and $C$ are similarly shaped (oval and rounded, respectively); thus, the evaluated parameters were affected mainly by snare stiffness. Product $B$, which is hexagonal and made of thin wire, had greater tightening force than did product $A$ with its oval shape and thick wire, thus logically reflecting the market's subjective estimation (good capture ability). Thus, these data suggest that our system reflects usability in vivo and reflect snare performance according to characteristics such as stiffness and shape. However, further studies regarding how our system benefits consumers are warranted.

We developed a novel system, but further steps are needed to show its true usefulness. First, additional existing snares should be evaluated using our system to assess consistency between our objective data and subjective usability in vivo. By confirming our results, objective methods of snare evaluation could be established in a stepwise manner, and the need for animal experimentation might be reduced in the future. In addition, the usable range of each parameter should be estimated according to the measured parameters, which may help in developing novel effective snares. Furthermore, a dynamic evaluation system for the cutting force, which is important for cold polypectomy, should also be developed.

\section{Conclusion}

In summary, we developed a novel system that may help objectively and "dynamically" evaluate polypectomy snares. Further experiments using our system are warranted.

\section{Acknowledgements}

The authors are very grateful to the Project ENGINE members for their collaboration on this work. They thank Traci Raley, MS, ELS, from Edanz Group (www.edanzediting.com/ac) for editing a draft of this manuscript.

\section{Competing interests}

This study was funded by a 2016-2017 strategic basic technology advancement support project, Ministry of Economy, Trade and Industry, Japan

\section{References}

[1] Winawer S], Zauber AG, Ho MN et al. Prevention of colorectal cancer by colonoscopic polypectomy. The National Polyp Study Workgroup. N Engl J Med 1993; 329: 1977-1981

[2] Rex DK, Pohl H. Considering the impact of the flexible polypectomy snare. Endoscopy 2019; 51: 109-110

[3] Ferlitsch M, Moss A, Hassan C et al. Colorectal polypectomy and endoscopic mucosal resection (EMR): European Society of Gastrointestinal Endoscopy (ESGE) Clinical Guideline. Endoscopy 2017; 49: 270-297

[4] Kawamura T, Takeuchi Y, Asai S et al. A comparison of the resection rate for cold and hot snare polypectomy for $4-9 \mathrm{~mm}$ colorectal polyps: a multicentre randomised controlled trial (CRESCENT study). Gut 2018; 67: 1950-1957

[5] Yamashina T, Uedo N, Akasaka T et al. Comparison of underwater vs conventional endoscopic mucosal resection of intermediate-size colorectal polyps. Gastroenterology 2019; 157: 451-461

[6] Yamasaki Y, Uedo N, Takeuchi Y et al. Underwater endoscopic mucosal resection for superficial nonampullary duodenal adenomas. Endoscopy 2018; 50: 154-158

[7] Shichijo S, Takeuchi Y, Kitamura M et al. Does cold snare polypectomy completely resect the mucosal layer? A prospective single-center observational trial J Gastroenterol Hepatol 2020; 35: 241-248

[8] Yamano H, Matsushita H, Yamanaka K et al. A study of physical efficacy of different snares for endoscopic mucosal resection. Digestive Endoscopy 2004; 16: S85-S88

[9] Russell WMS, Burch RL. The principles of humane experimental technique. Wheathampstead (UK): Universities Federation for Animal Welfare; 1959

[10] World Medical Association. World Medical Association Declaration of Helsinki: ethical principles for medical research involving human subjects. JAMA 2013; 310: 2191-2194 